\title{
Toward a harmonized and standardized protocol for the determination of total hydroxytyrosol and tyrosol content in virgin olive oil (VOO). Extraction solvent
}

Running title: Standardizing VOO polar phenol extraction solvent

Nikolaos Nenadis ${ }^{1}$, Aspasia Mastralexi ${ }^{1}$, Maria Z. Tsimidou ${ }^{1 *}$, Stefania Vichi $^{2}$, Beatriz

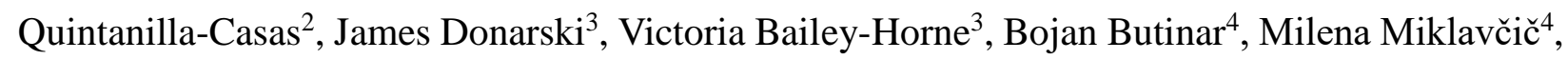
Diego-Luis García González ${ }^{5}$ Tullia Gallina Toschi ${ }^{6}$

${ }^{1}$ Laboratory of Food Chemistry and Technology, School of Chemistry, Aristotle University of Thessaloniki (AUTH), 541 24, Thessaloniki, Greece

${ }^{2}$ Food Science and Nutrition Department, XaRTA (Catalonian Reference Network on Food Technology), University of Barcelona (UB), Food and Nutrition Torribera Campus, Av. Prat de la Riba, 171. 08028, S.ta Coloma de Gramenet, Spain

${ }^{3}$ FERA Science Ltd, (FERA) Sand Hutton, York, YO41 1LZ, UK

${ }^{4}$ Laboratory of the Institute for Oliveculture, Science and Research Centre Koper (ZRS), Zelena ulica 8k, SI-6310 Izola, Slovenia

${ }^{5}$ Instituto de la Grasa (CSIC), Ctra. de Utrera, km. 1, Campus Universitario Pablo de Olavide Building 46, 41013 Seville, Spain

${ }^{6}$ Department of Agricultural and Food Sciences, Alma Mater Studiorum, University of Bologna (UNIBO), piazza Goidanich, 60, I-47521, Cesena (FC), Bologna, Italy

Corresponding author: Professor Maria Z. Tsimidou, Laboratory of Food Chemistry and Technology, School of Chemistry, Aristotle University of Thessaloniki (AUTH), 541 24, Thessaloniki, Greece, e-mail: tsimidou@chem.auth.gr; Tel: +302310997796 Fax: +30 2310 997847 
Abstract: The determination of the total hydroxytyrosol (Htyr) and tyrosol (Tyr) content of virgin olive oil is of utmost interest for the International Olive Council (IOC), food authorities, producers and distributors after the issuing of a health claim that 'olive oil polyphenols contribute to the protection of blood lipids from oxidative stress'. To address the need of a harmonized and standardized protocol the present study focuses on the extraction solvent of the polar fraction. Aqueous mixtures of methanol and acetonitrile of equal polarity were prepared and comparatively examined. Different analytical approaches (Folin-Ciocalteu assay, reversed phase chromatography-diode array-fluorescence detection, LC-Orbitrap, LC-TOF, LC-TQd, ${ }^{1} \mathrm{H}-\mathrm{NMR}$ ) were applied to highlight the extracting efficiency of the tested mixtures regarding the phenolic content and composition. The use of acetonitrile had not a clear positive effect that could compensate its higher cost, commercial availability and toxicity. The findings justify further why methanol:water, 80:20 v/v should be retained in a future IOC protocol for the accurate and repeatable determination of total Htyr and Tyr content, which is necessary to support the health claim for 'olive oil polyphenols'.

Practical applications: The development of a harmonized and standardized methodology for the determination of total Htyr and Tyr content in an explicit manner is a request of the olive oil sector. A tool that can be introduced easily in the olive industry and official laboratories for the control of the label that bears a health claim for 'olive oil polyphenols,' is also requested by the consumers and the IOC. In this view, the present study contributes to the cornerstone that is the standardization of the extraction solvent.

Keywords: Virgin olive oil phenols/ liquid-liquid extraction/ health claim/ total hydroxytyrosol and tyrosol/ standardization 
Abbreviations: ACN, acetonitrile; CA, caffeic acid; DA, diode array; DHPA, dihydroxyphenylacetic acid; DiMeA, dimethyl acetal; ESI, electrospray ionization; Ет(30), solvent polarity parameter; F-C, Folin Ciocalteu; FL, fluorescence; FWHM, full width at half maximum; H-ESI, heated electrospray ionization; HRMS, high resolution mass spectrometer; Htyr, hydroxytyrosol; IOC, International Olive Council; MeHA, methyl hemiacetal; MeOH, methanol; MRM, multiple reaction monitoring; $\mathbf{P}^{\prime}$, polarity index; QTOF, quadrupole time of flight; TOF time of flight; TPC, total phenol content; TQd, triple quadrupole; Tyr, tyrosol; VOO, virgin olive oil

\section{Introduction}

Hydroxytyrosol (Htyr) and tyrosol (Tyr) in their free and bound forms are the most abudant polar phenols of VOO. ${ }^{[1]}$ They contribute to its high resistance to oxidation, to various health benefits associated with its frequent consumption such as the protection of low-density lipoprotein particles from oxidative damage. ${ }^{[2-4]}$ The bound forms are responsible for the bitterness and astringency of the fresh product. ${ }^{[4]}$ The concentration and profile of these compounds depends on the cultivar, agricultural practices, fruit maturity and integrity, processing parameters and storage length and conditions all of which impact the commercial quality and claimed nutritional and health benefits of VOO. Therefore, since the late 60's much effort was devoted to the analysis of these compounds including the step of their extraction from the oil matrix..$^{[1,4-7]}$ The polar phenolic compounds are determinedin the 'polar fraction' of the oil, which is obtained with the aid of polar solvents. Evidence built up over the years indicates that liquid-liquid extraction is more efficient than solid phase one for this purpose. ${ }^{[4]}$ Aqueous mixtures of methanol $(\mathrm{MeOH})$, mainly $\mathrm{MeOH}: \mathrm{H}_{2} \mathrm{O}\left(80: 20 \mathrm{v} / \mathrm{v}\right.$ and 60:40 v/v), are used in the majority of applications, ${ }^{[7]}$ whereas, lately, the use of acetonitrile (ACN) is also discussed because - being a non-protic solvent - does not lead to the formation of artifacts as it is expected for $\mathrm{MeOH}$. In addition, its use seems to be beneficial for the recovery of the complex forms of Htyr and Tyr. ${ }^{[8,9]}$ Still, literature search among the 
numerous papers on the analysis of olive oil polar phenolic compounds indicates the need for standardization of this critical step.

In the frame of OLEUM project, seeking for the harmonization of procedures that can then be adopted in the analysis of olive oil for both regulatory and research purposes ${ }^{[10]}$ a systematic work to standardize step by step a procedure for the determination of total Htyr and Tyr content is undertaken. In particular, this study addresses the effect of the polar phenol extraction solvent. Such a work is of utmost interest for IOC, European food authorities, producers and distributors ${ }^{[11,}$ ${ }^{12]}$ after the issuing of a health claim that 'olive oil polyphenols contribute to the protection of blood lipids from oxidative stress'. ${ }^{[13]}$ For the aim of the study, aqueous mixtures of $\mathrm{MeOH}$ and ACN were comparatively examined as extraction solvents of the polar fraction. Ethanol, which is sometimes also reported, ${ }^{[14,15]}$ was not included as it exerts similar selectivity to $\mathrm{MeOH}$ and it is more expensive than that. The extraction solvent mixture used in the protocol that has been adopted by the IOC for the determination of biophenols in olive oil by HPLC ${ }^{[16]}$ was the reference point for our efforts to standardize this step. Several analytical methods (Folin Ciocalteu, HPLC-DA-FL, ${ }^{1} \mathrm{H}-\mathrm{NMR}$, LC-MS) have been applied to evaluate the recovery of phenolic compounds using the selected extraction solvent mixtures and to verify the presence/absence of artifacts.

\section{Materials and methods}

\subsection{Chemicals}

Tyr (98\%) and 1,3,5-triazine (97\%) were products of Alfa Aesar GmdH \& Co KG, (Karlsruhe, Germany). Caffeic acid (CA, 98\%) and 3,4-dihydroxyphenylacetic acid (DHPA, 98\%) were purchased from Sigma-Aldrich Chemie GmbH (Steinheim, Germany). Htyr (98\%) was from Extrasynthèse (Genay, France). $\mathrm{H}_{2} \mathrm{O}-\mathrm{d}_{2}$, (99.9\%) and DMSO- $\mathrm{d}_{6},(99.8 \%)$ were obtained from Deutero GmdH (Kastellaun, Germany). For mass spectrometry analysis, hexane and methanol of 
mass spectrometric grade (MS SupraSolv®) and formic acid (Suprapur®) were purchased by Merck (Darmstadt, Germany). Water was of ultrapure Milli-Q grade. Nitrogen (Alphagaz N2, purity $99.999 \%$, Air Liquid) was used in the Orbitrap as nebulization and fragmentation gas. Other reagents and solvents of appropriate grade were purchased from various producers.

\subsection{Virgin olive oil samples}

The VOO samples examined (S1-S30) were obtained directly from various producers and locations belonged to AUTH-OLEUM collection. Other details are given in the respective Tables and Figures.

\subsection{Preparation of the polar fraction}

\subsubsection{Calculation of the extraction solvent mixture composition}

The composition of aqueous ACN mixtures of equal polarity index values to the aqueous $\mathrm{MeOH}$ ones was calculated from the equation $P^{\prime}=\varphi a \times P a+\varphi b \times P b$, where $\varphi a$ and $\varphi b$ is the volume fraction of each solvent in the binary mixture and $P a, P b$ refers to $P^{\prime}$ values of the pure solvents. The $P^{\prime}$ values for $\mathrm{MeOH}(5.1), \mathrm{ACN}(5.8)$ and $\mathrm{H}_{2} \mathrm{O}$ (10.2) were from Snyder et al. ${ }^{[17]}$ Similarly, the composition of aqueous $\mathrm{ACN}$ mixtures of equal polarity $\mathrm{E}_{\mathrm{T}}(30)$ value to the aqueous $\mathrm{MeOH}$ ones was calculated from the equation $\mathrm{E}_{\mathrm{T}}(30)=63.0412-0.1773 \times(\% \mathrm{ACN})+0.0010 \times(\% \mathrm{ACN})^{2}$ using the $\mathrm{E}_{\mathrm{T}}(30)$ values provided by Dorsey and Johnson. ${ }^{[18]}$ According to the authors the equation is valid for $\mathrm{ACN}: \mathrm{H}_{2} \mathrm{O}$ mixtures in the range $0-80 \% \mathrm{v} / \mathrm{v}$.

\subsubsection{Extraction procedure}

The extraction procedure was as follows unless otherwise stated in the experimental, discussion, figures or tables: An aliquot of VOO (2.5 g) was dissolved in $5 \mathrm{~mL}$ of hexane and the polar fraction was extracted using an equal volume of the tested solvent. The mixture was vortexed for 2 min and centrifuged for $10 \mathrm{~min}$ at $3500 \mathrm{rpm} .{ }^{[19]}$ The extraction was carried out once after examination of the repeatability over the period of the last four years by different analysts $(C V \%=1.1-5.1 \%, n=5)$ 
using the Folin-Ciocalteu (F-C) assay.

\subsection{Colorimetric assessment of the total polar phenol content (TPC)}

Suitable aliquots of the polar extracts were transferred in a $10 \mathrm{~mL}$ volumetric flask and, subsequently, water $(5 \mathrm{~mL})$ and the Folin-Ciocalteu reagent $(0.5 \mathrm{~mL})$ were added. After $3 \mathrm{~min}, 1$ $\mathrm{mL}$ of saturated $(37 \%, \mathrm{w} / \mathrm{v})$ sodium carbonate solution was added to the reaction mixture. The solution was diluted with water to $10 \mathrm{~mL}$ and after $1 \mathrm{~h}$ the absorbance at $725 \mathrm{~nm}$ was measured against a blank solution with a spectrophotometer UV-1601 (Shimadzu Co., Kyoto, Japan). Caffeic acid (CA) was used as an external standard ${ }^{[19]}$ and results were expressed as $\mathrm{mg}$ CA $/ 20 \mathrm{~g}$ of oil. The determination was performed in triplicate for each extract (the repeatability over the period of the last four years by different analysts was $C V \%=1.1-2.7 \%, n=5$ ).

\subsection{Acidic hydrolysis of the polar fraction}

An aliquot $(200 \mu \mathrm{L})$ of the polar fraction was mixed with $200 \mu \mathrm{L}$ of a $1 \mathrm{M} \mathrm{H}_{2} \mathrm{SO}_{4}$ solution. The mixture was incubated in a water bath at $80{ }^{\circ} \mathrm{C}$ for $2 \mathrm{~h}^{[14]}$ The procedure was carried out in triplicate. Each hydrolysate was then diluted with $200 \mu \mathrm{L}$ of the polar fraction extracting solvent. The three replicates were combined to obtain a representative hydrolysate. The latter was filtered through a $0.45 \mu \mathrm{m}$ pore size regenerated cellulose membrane (Schleicher and Schuell, MicroScience GmbH, Dassel, Germany) before injection onto the chromatograph. ${ }^{[19]}$ Analysis was carried out in triplicate. The intra and interday repeatability of hydrolysis for Htyr and Tyr determination were $(C V \%)$ 0.9/2.4 and 1.5/2.2 ( $n=5)$, respectively.

\subsection{RP-HPLC analysis of the polar phenolic compounds}

The HPLC system at AUTH was consisted of a pump, model P4000 (Thermo Separation Products, San Jose, CA, USA), a Midas autosampler (Spark, Emmen, The Netherlands), and a UV 6000 LP diode array (DA) detector (Thermo Separation Products) in series with an SSI 502 fluorescene (FL) 
detector (Scientific Systems Inc., State College, PA, USA). Phenolic compounds in the tested extracts were monitored at $280 \mathrm{~nm}$ using DA and at $280 \mathrm{~nm}$ excitation and $320 \mathrm{~nm}$ emission using FL detection. The data were processed with the aid of Chrom Quest software (version 3.0, Thermo Separation Products). Analysis was carried out on a Nucleosil 100, C18 $(250 \times 4.6 \mathrm{~mm}, 5 \mu \mathrm{m})$ column (MZ-Analysentechnik GmbH, Mainz, Germany). The elution solvent was consisted of 1\% aqueous acetic acid (solvent $\mathrm{A}$ ) and $\mathrm{ACN}$ (solvent $\mathrm{B}$ ). The gradient used was: $0-2 \mathrm{~min}, 5 \% \mathrm{~B} ; 2-$ $15 \min , 25 \% \mathrm{~B} ; 15-22 \min , 25 \% \mathrm{~B} ; 22-30 \min , 40 \% \mathrm{~B} ; 30-40 \min , 60 \% \mathrm{~B} ; 40-50 \min , 95 \%$ $\mathrm{B} ; 50-52 \mathrm{~min}, 95 \% \mathrm{~B} ; 52-54 \mathrm{~min}, 100 \% \mathrm{~B} ; 54-60 \mathrm{~min}, 5 \% \mathrm{~B}$, at a flow rate of $0.5 \mathrm{~mL} / \mathrm{min}$ and an injection volume of $10 \mu \mathrm{L} .{ }^{[19]}$ In certain experiments (section 3.2) the separation was carried out using the gradient proposed by $\mathrm{IOC}^{[16]}$ or with slight modifications. Quantitative data using various standards were expressed as $\mathrm{mg}$ or $\mu \mathrm{mol} / 20 \mathrm{~g}$ oil.

\subsection{LC-MS analysis}

LC-MS analysis of the same samples was carried out at 3 different laboratories, each one having different MS facilities within a specific timetable ( 2 months): (i) an LC system consisted of a Vanquish pump and autosampler (Thermo Scientific - Dionex Softron, Germering, DE), coupled to a Q-Exactive hybrid Orbitrap mass spectrometer (Thermo Fisher Scientific, Bremen, Germany), which was equipped with an electrospray source (H-ESI II) at UB; (ii) a 1200 Series LC and a 6230 time-of-flight (TOF) mass spectrometer (Agilent, Santa Clara, CA, USA) at FERA and (iii) an Infinity 1260 HPLC (Agilent Technologies, Waldbronn, Germany) consisted of a G1312B binary pump, a G4225 degasser, a G1329B ALS, a G1330B thermostat, a G1316A thermostated column compartment and a G4212B DA detector (190-600 nm) interfaced with a triple-quadrupole (TQd) mass spectrometer (Triple Quad G6420A LC/MS; Agilent Technologies, Singapore) at ZRS. Details on the elution conditions adjusted to each MS instrument are provided as supplementary material (Text $\mathrm{S} 1)$. 


\section{8. ${ }^{1} \mathrm{H}-\mathrm{NMR}$ analysis}

${ }^{1} \mathrm{H}-\mathrm{NMR}$ experiments were conducted on a $500 \mathrm{MHz}$ NMR (Agilent Technologies Inc., Santa Clara, CA). The probe temperature was $25^{\circ} \mathrm{C}$. All chemical shifts were given in ppm from TMS $(\delta$ 0.00). For quantitative ${ }^{1} \mathrm{H}-\mathrm{NMR}$ measurements, the experimental parameters were: repetition time (acquisition time + relaxation delay) 12s, 32k data points, number of scans 32 with 4 dummy scans. Baseline correction (applying a polynomial fourth-order function) and phasing were carried out manually prior to the integration process using appropriate software (Mestrelab Research Lab, ver. 6.0.2-5475, 2009, La Coruña, Spain). The preparation of the polar fraction using $\mathrm{MeOH}: \mathrm{H}_{2} \mathrm{O}$, 80:20, v/v, $\mathrm{MeOH}: \mathrm{H}_{2} \mathrm{O}, 60: 40$, v/v or $\mathrm{ACN}: \mathrm{H}_{2} \mathrm{O}, 70: 30$, v/v, and total Htyr and Tyr determination in the polar fraction was carried out according to Dais and Christophoridou. ${ }^{[20]}$ The results were expressed as $\mu \mathrm{mol} / 20 \mathrm{~g}$ of oil. The $C V \%$ of the measurement for a VOO sample was $0.6(n=5)$.

\subsection{Statistical analysis}

Statistical comparisons of the mean values were performed by one-way ANOVA, followed by the multiple Duncan test ( $p<0.05$ confidence level) using the SPSS 14.0 software (SPSS Inc., Chicago, IL, USA). The same software was used for pairwise student's t-test comparison of means at $p<0.05$ where necessary.

\section{Results and discussion}

\subsection{Selection of aqueous acetonitrile composition}

$\mathrm{MeOH}$ and $\mathrm{ACN}$ are two polar solvents of different selectivity characteristics (Table S1) ${ }^{[17,21]}$ so that the study of their extracting efficiency at equal percentages in aqueous mixtures ${ }^{[22]}$ is not the most appropriate one. In the present study selection of the most efficient aqueous ACN mixture with regard to the performance of the aqueous $\mathrm{MeOH}$ mixture proposed by IOC, namely $\mathrm{MeOH}: \mathrm{H}_{2} \mathrm{O}, 80: 20 \mathrm{v} / \mathrm{v}$, was, thus, based on different selectivity criteria and not on percentages. 
The aqueous ACN mixtures tested had a composition of comparable polarity to those of the $\mathrm{MeOH}$ one using either the Snyder polarity index $\left(P^{\prime}\right)^{[17]}$ or the solvent polarity parameter $\mathrm{E}_{\mathrm{T}}(30) .{ }^{[18]}$ The $P^{\prime}$ is a measure of solvent ability to interact with solutes of different functionality, ${ }^{[17]}$ whereas $\mathrm{E}_{\mathrm{T}}(30)$ is a descriptor of both hydrogen bond and electrostatic interactions of the solvent. ${ }^{[23]}$ The percentages were calculated as described in section 2.3.1. The mixtures examined were, consequently, $\mathrm{ACN}: \mathrm{H}_{2} \mathrm{O} 93: 7, \mathrm{v} / \mathrm{v}\left(P^{\prime}\right.$ index $)$ and 51:49, v/v $\left(\mathrm{E}_{\mathrm{T}}(30)\right.$ parameter). Moreover, the mixtures 70:30 v/v ( $P^{\prime}$ index $)$ and 39:61, v/v ( $\mathrm{E}_{\mathrm{T}}(30)$ parameter) corresponding to the polarity of the $\mathrm{MeOH}: \mathrm{H}_{2} \mathrm{O}, 60: 40$, v/v were tested to highlight further the overall performance differences between the two organic solvents. The tests were performed on a VOO (S1) containg both free and bound forms of Htyr and Tyr because it was a blend of freshly produced oil and of an oil stored at room temperature for about one year in the dark. Estimation of the solvent efficiency was evaluated in terms of F-C assay and HPLC determination of the TPC. Pure ACN and its 93\% aqueous mixture extracted lipids and pigments that are expected to interfere in subsequent analyses, colorimetric or liquid chromatographic, so that they were rejected as options. From the rest of the combinations of $\mathrm{ACN}: \mathrm{H}_{2} \mathrm{O}$ mixtures the most efficient was the $70: 30$, v/v one with reference to the IOC proposed solvent (Table 1). The latter was in turn more efficient than the $\mathrm{MeOH}: \mathrm{H}_{2} \mathrm{O}, 60: 40, \mathrm{v} / \mathrm{v}(p<0.05)$. The same trend was observed by both the colorimetric and chromatographic analysis. These observations were verified for 5 more VOOs (S2-S6) randomly picked up from the AUTH OLEUM collection (Table 2).

Information from Table 2 together with that obtained for the total Htyr and Tyr content determined using ${ }^{1} \mathrm{H}-\mathrm{NMR}$ spectroscopy (Table 3) gave a strong evidence that further examination of the $\mathrm{MeOH}: \mathrm{H}_{2} \mathrm{O}, 60: 40$, v/v mixture was not promising for the aim of a standardised protocol. The other two solvent systems deserved further examination.

\section{2. $\mathrm{MeOH}: \mathrm{H}_{2} \mathrm{O}, 80: 20$, v/v, vs $\mathrm{ACN}: \mathrm{H}_{2} \mathrm{O}, 70: 30$, v/v mixtures as extraction solvents}

The VOO polar fraction is used for the estimation of the total content or individual phenol before or after hydrolysis, mainly using RP-HPLC. ${ }^{[5,15,16,19,24-26]}$ Data shown in Figure 1 for the TPC (mg 
CA /20 g oil) for twenty-one (21) authentic VOOs (S7-S27) extracted with the two prevailing solvent mixtures indicated that aqueous ACN gave statistically significant higher values only in five samples. In particular, these values were only 1.1-1.3-fold higher, suggesting that the two solvent mixtures are of equivalent potency. Further insight in quantitative and qualitative differences was sought using RP-HPLC-DA-FL and LC-MS analyses.

\subsubsection{RP-HPLC-DA-FL analysis}

Introduction of the polar extract to the liquid chromatograph is usually carried out after drying and redilution in a volume of $\mathrm{MeOH}: \mathrm{H}_{2} \mathrm{O}$ or $\mathrm{MeOH}$ even if in the gradient elution ACN is used. ${ }^{[19,22 \text {, }}$ 24, 25, 27] In the IOC recommended protocol, the polar fraction is injected directly onto the HPLC system probably to avoid further sample manipulation, which may increase the labour and time of analysis. There is no published paper that details all the steps of the development of this protocol which has been accepted after a collaborative study and data evaluation according to ISO 5725. ${ }^{[16]}$ Seeking for differences between the two extraction solvent mixtures, the VOOs S10, S13, S17, S19 and S24 were also examined using this protocol. The samples were of the same cultivar and location and contained almost comparable TPC by F-C. The choice aimed at reducing the effect of cultivar, geographical origin and magnitude of content among findings. The profiles recorded using DA and FL detectors are given in Figure 2. Quantification was carried out before (TPC) and after hydrolysis (total Htyr and Tyr content) at $280 \mathrm{~nm}$. Different standard curves or result expression were used. The quantitative results are presented in Table 4.

From the recorded profiles of the two extracts it was observed that they were qualitatively similar using either DA or FL detection. The F-C findings (see Figure 1) were not reflected in the chromatograms but for all pairs of samples -regardless of the standard used for quantification of the TPC- the values obtained for the aqueous $\mathrm{MeOH}$ extracts differed statistically from those of the aqueous ACN ones (Table 4). Using Htyr for quantification, lower values (1.67-1.70 fold) than 
those acquired with Tyr as a standard were obtained. These values were only 1.02-1.07 fold higher than those expressed as DHPA equivalents. The latter phenolic acid was selected as a possible alternative to Htyr considering the structural similarity and its lower cost. Despite the fluctuation in the absolute values, the use of different standards did not augment or suppress the relative differences. As a consequence, the findings led to similar judgments. Focusing on total Htyr and Tyr content by hydrolysis of the polar fraction, statistical significant differences were observed in three of the samples for both constituents. Nevertheless, no clear superiority of one solvent mixture over the other was observed regardless of the result expression.

\subsubsection{LC-MS analysis}

Three pairs of polar phenol extracts from samples S28, S29 and S30 were then prepared using MeOH: $\mathrm{H}_{2} \mathrm{O}, 80: 20$, v/v and $\mathrm{ACN}: \mathrm{H}_{2} \mathrm{O}, 70: 30$, v/v. Their profiles recorded at $280 \mathrm{~nm}$ (Figure S1) were qualitatively similar. Then, they were further studied by different LC-MS facilities, to unravel, mainly, any qualitative differences. In all cases the organic solvent of the gradient was MeOH:ACN, 1:1, v/v as in the IOC protocol. ${ }^{[16]}$ The ESI $^{+}$and ESI ${ }^{-}$full scan chromatograms of the extracts in the mass range $m / z$ 315-395 (Figures 3 and 4, respectively) obtained by the LC-Orbitrap mass spectrometer showed no qualitative differences. This was further verified for the main bound forms on the basis of the extracted ion chromatograms using their exact mass as presented in the same Figures. Only quantitative differences were observed. Thus, aqueous ACN extraction favored some compounds eluting at the end of the chromatogram (e.g. $\mathrm{t}_{\mathrm{R}}=18.10, \mathrm{~m} / \mathrm{z} 377.1242$ $\left.[\mathrm{M}-\mathrm{H}]^{-}, \mathrm{C}_{19} \mathrm{H}_{21} \mathrm{O}_{8} ; \mathrm{t}_{\mathrm{R}}=20.23, \mathrm{~m} / z 361.1293[\mathrm{M}-\mathrm{H}]^{-}, \mathrm{C}_{19} \mathrm{H}_{21} \mathrm{O}_{7}\right)$. On the other hand, aqueous MeOH extraction favored some more polar derivatives (e.g. $\mathrm{t}_{\mathrm{R}}=12.82,13.33 \mathrm{~m} / \mathrm{z} 377.1242[\mathrm{M}-\mathrm{H}]^{-}$, $\left.\mathrm{C}_{19} \mathrm{H}_{21} \mathrm{O}_{8} ; \mathrm{t}_{\mathrm{R}}=14.92,15.49, \mathrm{~m} / \mathrm{z} 361.1293[\mathrm{M}-\mathrm{H}]^{-}, \mathrm{C}_{19} \mathrm{H}_{21} \mathrm{O}_{7}\right)$. The evidence that aqueous ACN favored the extraction of less polar compounds was verified by LC-TOF $\left(\mathrm{t}_{\mathrm{R}}=15.054 \mathrm{~min}, \mathrm{~m} / \mathrm{z}\right.$ $361.1290[\mathrm{M}-\mathrm{H}]^{-} ; \mathrm{t}_{\mathrm{R}}=16.499 \mathrm{~min}, \mathrm{~m} / z 377.1237[\mathrm{M}-\mathrm{H}]^{-}$) (Figure S2). The hypothesis that artifacts due to the presence of $\mathrm{MeOH}$ in the extract could be detected was examined for oleacein and 
oleocanthal ones using also TQd at the MRM mode. ${ }^{[28,29]}$ Using all LC-MS facilities the methyl hemiacetal (MeHA) of the two phenols was detected in both type of extracts. Only with the aid of TQd at the MRM mode, which is the most sensitive type of experiment, the dimethylacetal (DiMeA) of oleocanthal was found in all the extracts, whereas DiMeA of oleacein in the two extracts only of S28. The artifacts eluted at the same retention time with that of the precursors. Furthermore, calculation of the relative concentration of the artifacts detected (Table S.2) on basis of the peak area recorded by the three MS platforms showed that their levels in both type of extracts per sample were always comparable (maximum difference $\leq 2 \%$ ). Such an observation indicates an effect of the mobile phase composition and not of the extraction system. Present findings are in agreement with those of Sánchez-de Medina et al. ${ }^{[29]}$ who analysing with LC-QTOF at MRM mode a pure $\mathrm{ACN}$ or a $\mathrm{MeOH}: \mathrm{H}_{2} \mathrm{O}, 60: 40$, v/v polar extract: (i) reported a similar \% formation of oleacein and oleocantal artifacts ( 16\%)- the MeHAs were the main artifacts-in both of them when the elution protocol had $\mathrm{MeOH}$ in the gradient composition; (ii) when the same extracts were analyzed using a gradient containing only ACN, the corresponding artifacts were not detected. Thus, using the IOC proposed HPLC protocol the presence of $\mathrm{MeOH}$ artifacts is unavoidable regardless of the extract solvent.

\section{Conclusion}

The systematic work proved to be useful toward harmonization and standardization of an extraction protocol for the polar fraction of olive oil, which then can be used for the determination of total polar phenols or of the total content of Htyr and Tyr. The use of ACN had not a clear positive effect that can compensate its higher cost, commercial availability and toxicity that could support its adoption in a standardized protocol. The findings of this work justify further why $\mathrm{MeOH}: \mathrm{H}_{2} \mathrm{O}$ should be retained in an IOC protocol for the accurate and repeatable determination of total Htyr and Tyr content necessary to support the health claim for 'olive oil polyphenols'. 
Artifacts are rather related to the presence of $\mathrm{MeOH}$ in the elution gradient but if the total Htyr and Tyr content is determined after hydrolysis of the polar fraction no effect in the quantitation should be expected.

\section{Acknowledgement}

This work was developed in the context of the project OLEUM 'Advanced solutions for assuring authenticity and quality of olive oil at global scale' funded by the European Commission within the Horizon 2020 Programme (2014-2020, grant agreement no. 635690).

\section{Conflict of interest}

The authors state that there is no conflict of interest

\section{References}

[1] A. Bendini, L. Cerretani, A. Carrasco-Pancorbo, A. M. Gómez-Caravaca, A. Segura-Carretero, A. Fernández-Gutiérrez, G. Lercker, Molecules 2007, 12, 1679.

[2] D. Boskou, M. Tsimidou, G. Blekas, in Olive oil Chemistry technology (Ed: D. Boskou), AOCS press, Champaign, Il 2006, Ch.5.

[3] F. Visioli, P. Bogani, C. Galli, in Olive oil Chemistry technology, (Ed: D. Boskou), AOCS press, Champaign Il 2006, Ch.8.

[4] M. Z. Tsimidou, in Handbook of olive oil: Analysis and properties, (Eds: R. Aparicio, J. Harwood), $2^{\text {nd }}$ edition, Springer Science + Business Media, NY, 2013, Ch.9.

[5] M. Tsimidou, Ital. J. Food Sci. 1998, 10, 99. 
[6] A. Segura-Carretero, A. Carrasco-Pancorbo, A. Bendini, L. Cerretani, A. Fernández-Gutiérrez, in Olives and olive oil in health and disease prevention, (Eds: $\mathrm{V}$. Preedy, R.R. Watson), Academic Press, Burlington, MA, 2010, Ch.56.

[7] A. M. Gómez-Caravaca, J. Lozano-Sánchez, M. D. M. Contreras Gámez, A. S. Carretero, A. Taamalli, in Olive and olive oil bioactive constituents, (Ed.: D. Boskou) AOCS Press, Urbana, IL, 2015, Ch.9.

[8] J. Impellizzeri, J. Lin, J. Agric. Food Chem. 2006, 54, 3204.

[9] E. Karkoula, A. Skantzari, E. Melliou, P. Magiatis, J. Agric. Food Chem. 2012, 60, 11696.

[10] Better solutions to protect olive oil quality and authenticity (http://www.oleumproject.eu/ )

[11] M. Z. Tsimidou, D. Boskou, Eur. J. Lipid Sci. Technol. 2015, 117, 1091.

[12] http://www.internationaloliveoil.org/documents/index/10-documentos/1394-fichas-quimic $\underline{\text { a-news } / 3}$

[13] EC Regulation No. 432/2012 of 16 May 2012 establishing a list of permitted health claims made on foods, other than those referring to the reduction of disease risk, to children's development, health. Off. J. Eur. Comm. 2012, L136, 1.

[14] N. Mulinacci, C. Giaccherini, F. Ieri, M. Innocenti, A. Romani, F. F. Vincieri, J. Sci. Food Agric. 2006, 86, 757.

[15] S. Alessandri, F. Ieri, A. Romani, J. Agric. Food Chem. 2014, 62, 826.

[16] IOC, COI/T.20/Doc. no. 29, November 2009.

[17] L. R. Snyder, J. J. Kirkland, J. W. Dolan, Introduction to Modern Liquid Chromatography; Wiley: Hoboken, NJ, 2010.

[18] J. G. Dorsey, B. P. Johnson, J. Liquid Chromatogr. 1987, 10, 2695.

[19] A. Mastralexi, N. Nenadis, M. Z. Tsimidou, J. Agric. Food Chem., 2014, 62, 2459 (C. Romero, M. Brenes, J. Agric. Food Chem. 2014, 62, 10210; A. Mastralexi, N. Nenadis, M. Z. Tsimidou, J. Agric. Food Chem. 2014, 62, 10212).

[20] S. Christophoridou, P. Dais, Anal. Chim. Acta 2009, 633, 283. 
[21] C. Reichardt, T. Welton, Solvents and Solvent Effects in Organic Chemistry, Wiley-VCH Verlag GmbH \& Co. KGaA, Weinheim, Germany, 2010.

[22] A. L. Capriotti, C. Cavaliere, C. Crescenzi, P. Foglia, R. Nescatelli, R. Samperi, a. Laganà, Food Chem. 2014, 158, 392.

[23] J. P. Cerón-Carrasco, D. Jacquemin, C. Laurence, A. Planchat, C. Reichardt, K.Sraïdi, J. Phys. Org. Chem. 2014, 27, 512.

[24] K. Hrncirik, S. Fritsche, Eur. J. Lipid Sci. Technol. 2004, 106, 540.

[25] R. García-Villalba, A. Carrasco-Pancorbo, C. Oliveras-Ferraros, A. Vázquez-Martín, J. A. Menéndez, A. Segura-Carretero, A. Fernández-Gutiérrez, J. Pharm. Biomed. Anal. 2010, $51,416$.

[26] P. Reboredo-Rodríguez, E. Valli, A. Bendini, G. Di Lecce, J. Simal-Gándara, T. Gallina Toschi, Eur. J. Lipid Sci. Technol. 2016, 118, 1593.

[27] F. M. Pirisi, P. Cabras, C. F. Cao, M. Migliorini, M. Muggelli, J. Agric. Food Chem. 2000, $48,1191$.

[28] R. J. Luján, F. P. Capote, A. Marinas, M. D. L. de Castro, Rapid Comm. Mass Spec. 2008, 22,855 .

[29] V. Sánchez de Medina, H. Miho, E. Melliou, P. Magiatis, F. Priego-Capote, M. D. L. de Castro, Talanta, 2017, 162, 24. 
Table 1. TPC of sample S1 estimated by F-C assay and RP-HPLC- DA (280 nm) or FL (exc $280 \mathrm{~nm} / \mathrm{em} 320 \mathrm{~nm}$ ) detection after extraction with different $\mathrm{MeOH}$ and ACN aqueous mixtures

\begin{tabular}{|c|c|c|c|}
\hline \multirow[b]{2}{*}{ Extraction solvent (v/v) } & F-C assay & HPLC-DA & HPLC-FL \\
\hline & \multicolumn{3}{|c|}{ TPC $($ mean value $\pm \mathrm{SD}, n=3$ ) $* * *$} \\
\hline MeOH: $\mathrm{H}_{2} \mathrm{O}, 80: 20$ & $3.18 \pm 0.12^{\mathrm{a}}$ & $2.15 \pm 0.02^{\mathrm{a}}$ & $1.33 \pm 0.01^{\mathrm{a}}$ \\
\hline $\mathrm{MeOH}: \mathrm{H}_{2} \mathrm{O}, 60: 40$ & $2.46 \pm 0.03^{\mathrm{b}}$ & $1.86 \pm 0.01^{\mathrm{b}}$ & $1.13 \pm 0.01^{\mathrm{b}}$ \\
\hline $\mathrm{ACN}: \mathrm{H}_{2} \mathrm{O}, 70: 30$ & $3.54 \pm 0.02^{\mathrm{c}}$ & $2.26 \pm 0.02^{\mathrm{c}}$ & $1.37 \pm 0.01^{\mathrm{c}}$ \\
\hline $\mathrm{ACN}: \mathrm{H}_{2} \mathrm{O}, 51: 49$ & $2.58 \pm 0.08^{\mathrm{b}, \mathrm{d}}$ & $2.00 \pm 0.01^{\mathrm{d}}$ & $1.29 \pm 0.00^{\mathrm{d}}$ \\
\hline $\mathrm{ACN}: \mathrm{H}_{2} \mathrm{O}, 39: 61$ & $2.50 \pm 0.11^{\mathrm{b}, \mathrm{d}}$ & $1.86 \pm 0.02^{\mathrm{b}}$ & $1.20 \pm 0.01^{\mathrm{e}}$ \\
\hline
\end{tabular}

*TPC values expressed as mg CA /20 g oil (F-C); mg Tyr $/ 20 \mathrm{~g}$ oil (HPLC- DA $280 \mathrm{~nm}$ and FLexc $280 \mathrm{~nm} / \mathrm{em} 320 \mathrm{~nm}$ ); **Values in the same column bearing different lower case letters as superscripts are statistically different $(p<0.05)$ 
Table 2. TPC of VOOs (S2-S6) estimated by the F-C assay and RP-HPLC- DA (280 nm) or FL (exc. $280 \mathrm{~nm} / \mathrm{em} .320 \mathrm{~nm}$ ) detection after extraction with different $\mathrm{MeOH}$ and $\mathrm{ACN}$ aqueous mixtures

VOOs

\begin{tabular}{ccccc}
\hline S2 & S3 & S4 & S6 \\
\hline & F-C assay \\
& S \\
\hline
\end{tabular}

Extraction

TPC

solvent $(\mathbf{v} / \mathbf{v})$

mean value $\pm \mathrm{SD}, n=3, \mathrm{mg} \mathrm{CA} / 20 \mathrm{~g}$ oil*

\begin{tabular}{|c|c|c|c|c|c|}
\hline $\mathrm{MeOH}: \mathrm{H}_{2} \mathrm{O}$ & $3.71 \pm 0.08^{\mathrm{a}}$ & $4.23 \pm 0.07^{\mathrm{a}}$ & $3.16 \pm 0.06^{\mathrm{a}}$ & $3.54 \pm 0.09^{\mathrm{a}}$ & $2.14 \pm 0.03^{\mathrm{a}}$ \\
\hline \multicolumn{6}{|l|}{ 60:40 } \\
\hline $\mathrm{MeOH}: \mathrm{H}_{2} \mathrm{O}$, & $4.23 \pm 0.07^{b}$ & $4.53 \pm 0.12^{\mathrm{b}}$ & $3.57 \pm 0.06^{\mathrm{b}}$ & $4.17 \pm 0.06^{\mathrm{b}}$ & $2.62 \pm 0.04^{b}$ \\
\hline \multicolumn{6}{|l|}{$80: 20$} \\
\hline $\mathrm{ACN}: \mathrm{H}_{2} \mathrm{O}$ & $4.08 \pm 0.03^{c}$ & $4.42 \pm 0.05^{\mathrm{b}}$ & $3.44 \pm 0.07^{b, c}$ & $3.97 \pm 0.07^{c}$ & $2.77 \pm 0.05^{\mathrm{c}}$ \\
\hline 70:30 & & & & & \\
\hline
\end{tabular}

\section{RP-HPLC DA/(FL)}

\section{TPC}

mean value $\pm \mathrm{SD}, n=3, \mathrm{mg}$ Tyr $/ 20 \mathrm{~g}$ oil*

\begin{tabular}{lccccc}
\hline $\mathrm{MeOH}: \mathrm{H}_{2} \mathrm{O}$, & $2.41 \pm 0.06^{\mathrm{a}}$ & $2.33 \pm 0.08^{\mathrm{a}}$ & $1.78 \pm 0.03^{\mathrm{a}}$ & $2.01 \pm 0.03^{\mathrm{a}}$ & $1.20 \pm 0.02^{\mathrm{a}}$ \\
$60: 40$ & $\left(0.77 \pm 0.01^{\mathrm{a}}\right)$ & $\left(1.47 \pm 0.05^{\mathrm{a}}\right)$ & $\left(0.73 \pm 0.01^{\mathrm{a}}\right)$ & $\left(1.0 \pm 0.00^{\mathrm{a}}\right)$ & $\left(0.57 \pm 0.01^{\mathrm{a}}\right)$ \\
$\mathrm{MeOH}: \mathrm{H}_{2} \mathrm{O}$, & $2.45 \pm 0.04^{\mathrm{a}}$ & $2.81 \pm 0.10^{\mathrm{b}}$ & $1.81 \pm 0.03^{\mathrm{b}}$ & $2.16 \pm 0.03^{\mathrm{b}}$ & $1.35 \pm 0.01^{\mathrm{b}}$ \\
$80: 20$ & $\left(0.80 \pm 0.00^{\mathrm{b}}\right)$ & $\left(1.73 \pm 0.05^{\mathrm{b}}\right)$ & $\left(0.82 \pm 0.02^{\mathrm{b}}\right)$ & $\left(1.07 \pm 0.01^{\mathrm{b}}\right)$ & $\left(0.58 \pm 0.00^{\mathrm{a}}\right)$ \\
$\mathrm{ACN}: \mathrm{H}_{2} \mathrm{O}$, & $2.46 \pm 0.04^{\mathrm{a}}$ & $2.85 \pm 0.01^{\mathrm{b}}$ & $1.92 \pm 0.04^{\mathrm{c}}$ & $2.32 \pm 0.03^{\mathrm{c}}$ & $1.41 \pm 0.02^{\mathrm{c}}$ \\
$70: 30$ & $\left(0.85 \pm 0.01^{\mathrm{c}}\right)$ & $\left(1.75 \pm 0.02^{\mathrm{b}}\right)$ & $\left(0.90 \pm 0.01^{\mathrm{c}}\right)$ & $\left(1.26 \pm 0.01^{\mathrm{c}}\right)$ & $\left(0.62 \pm 0.00^{\mathrm{b}}\right)$ \\
\hline
\end{tabular}

*Values in the same column, within each technique, bearing different lower case letters as superscripts are statistically different $(p<0.05)$ 
Table 3. Total Htyr and Try content of VOOs (S1-S6) determined by ${ }^{1} \mathrm{H}-\mathrm{NMR}$ after extraction with different $\mathrm{MeOH}$ and $\mathrm{ACN}$ aqueous mixtures

\begin{tabular}{|c|c|c|c|c|c|c|}
\hline \multirow{4}{*}{$\begin{array}{c}\text { Extraction solvent } \\
(\mathrm{v} / \mathrm{v})\end{array}$} & \multicolumn{6}{|c|}{ VOOs } \\
\hline & S1 & S2 & S3 & S4 & S5 & S6 \\
\hline & \multicolumn{6}{|c|}{ Total Htyr and Tyr } \\
\hline & \multicolumn{6}{|c|}{$\mu \mathrm{mol} / 20 \mathrm{~g}$ oil } \\
\hline $\mathrm{MeOH}: \mathrm{H}_{2} \mathrm{O}, 60: 40$ & 30.5 & 26.0 & 19.1 & 18.9 & 32.9 & 30.4 \\
\hline $\mathrm{MeOH}: \mathrm{H}_{2} \mathrm{O}, 80: 20$ & 33.1 & 27.0 & 27.3 & 24.0 & 42.0 & 31.3 \\
\hline $\mathrm{ACN}: \mathrm{H}_{2} \mathrm{O}, 70: 30$ & 34.4 & 28.0 & 28.1 & 25.8 & 37.8 & 32.6 \\
\hline
\end{tabular}


Table 4. TPC, total Htyr and Tyr content of 5 monovarietal VOOs determined by HPLC-DA (280 nm) after extraction with $\mathrm{MeOH}: \mathrm{H}_{2} \mathrm{O}$, $80: 20$, v/v or $\mathrm{ACN}: \mathrm{H}_{2} \mathrm{O}, 70: 30, \mathrm{v} / \mathrm{v}$

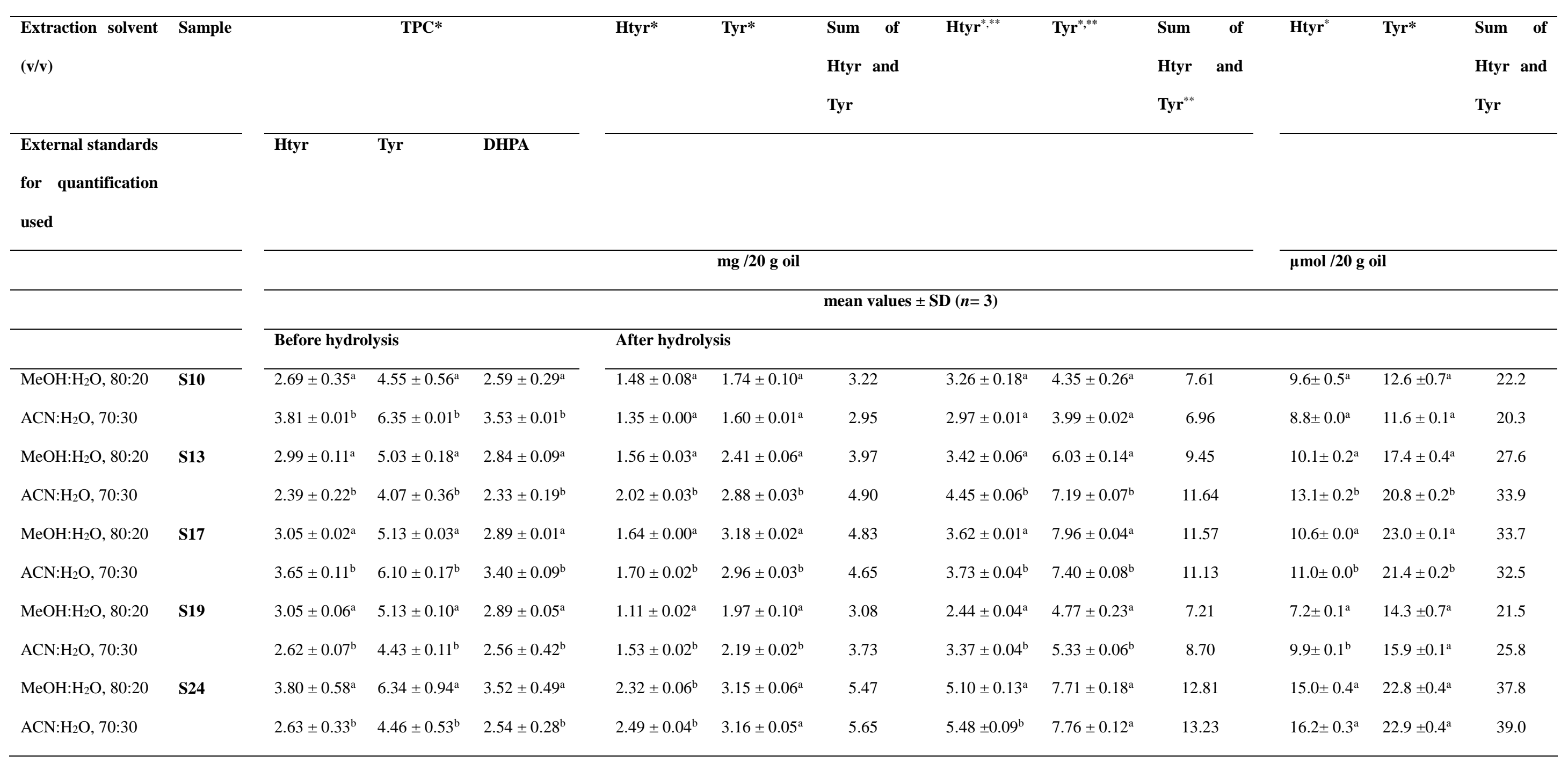

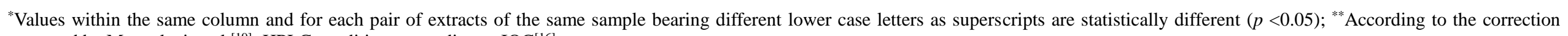
proposed by Mastralexi et al. ${ }^{[19]}$; HPLC conditions according to IOC ${ }^{[16]}$ 


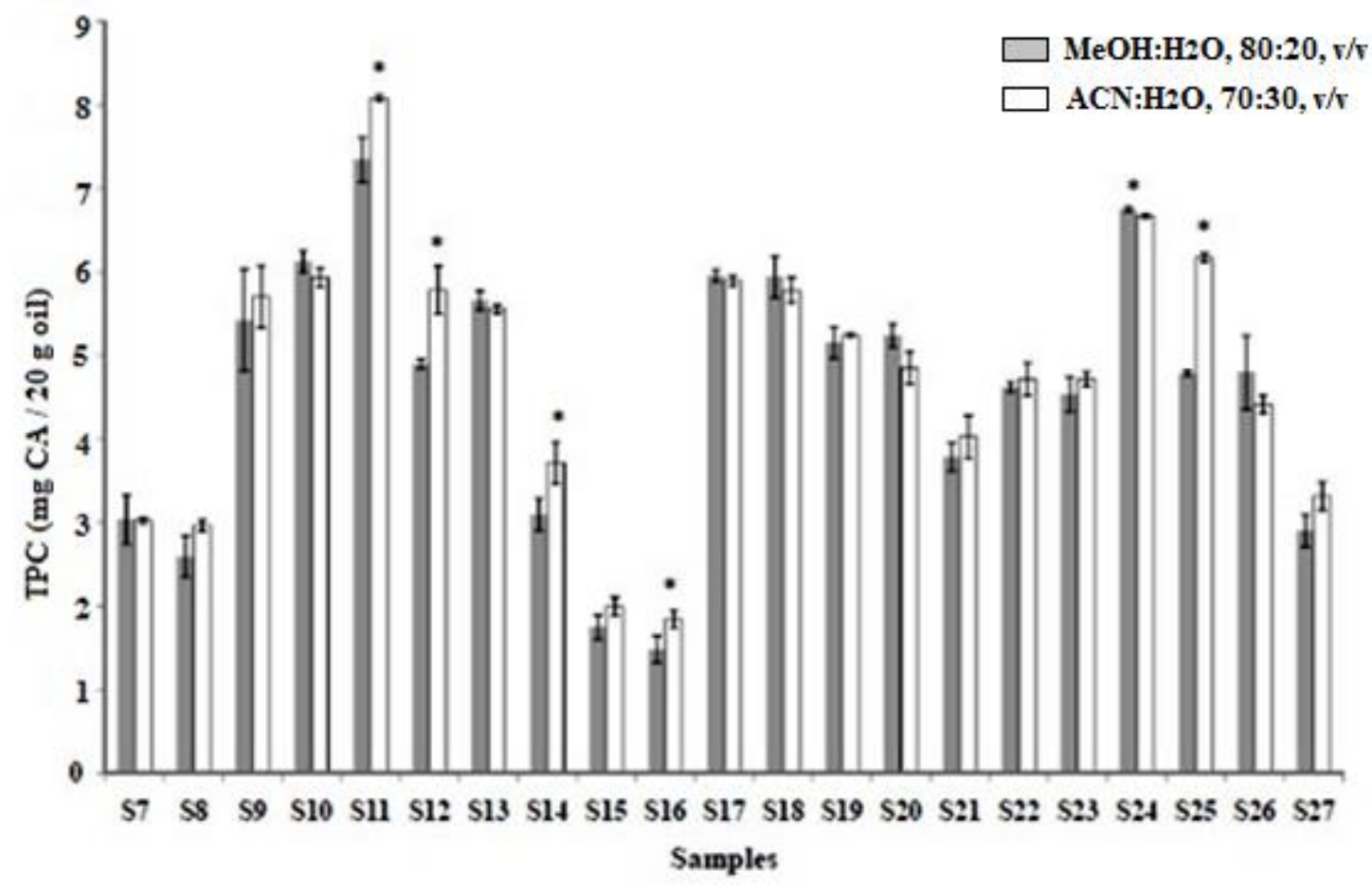

Figure 1 

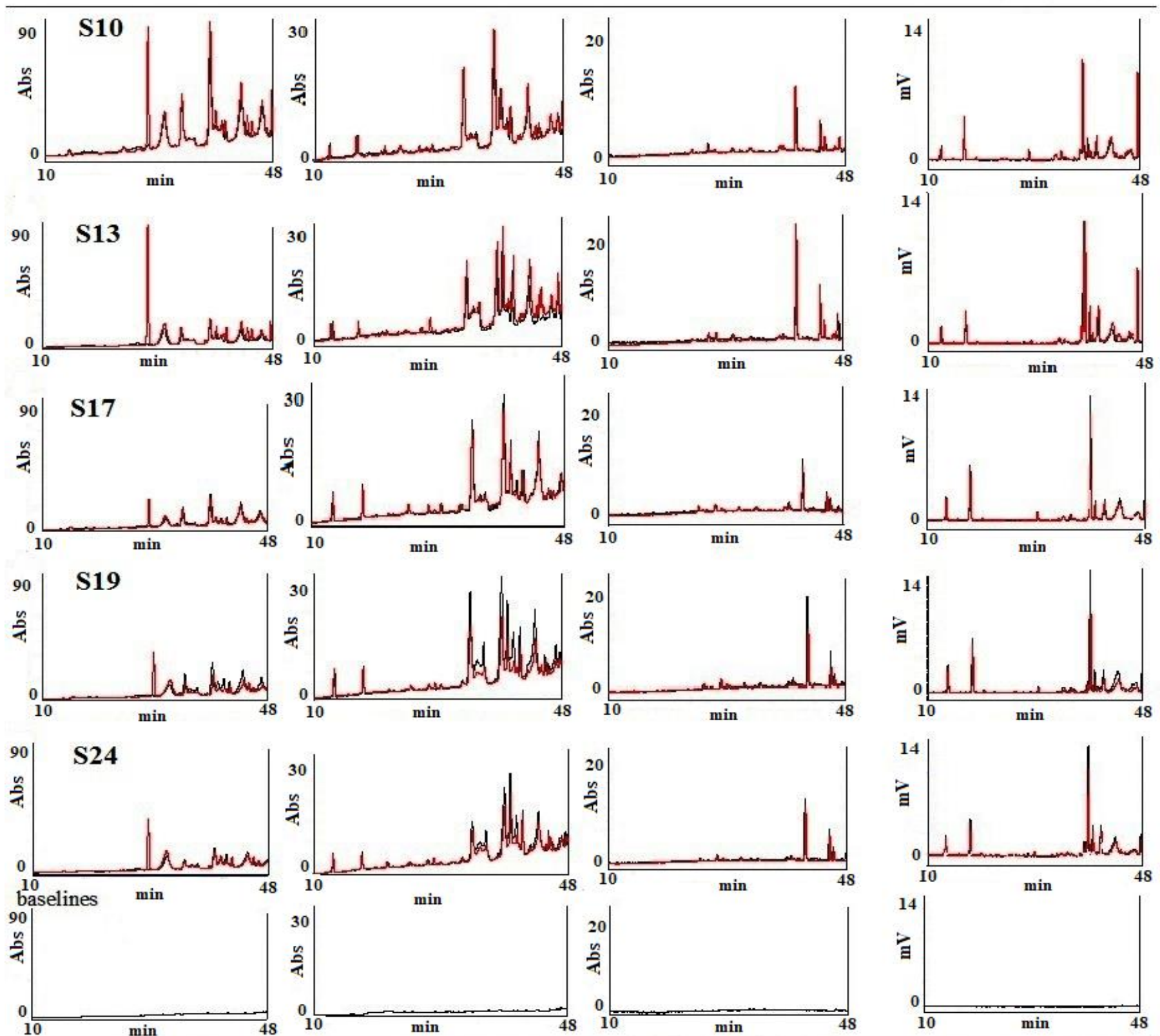

Figure 2 


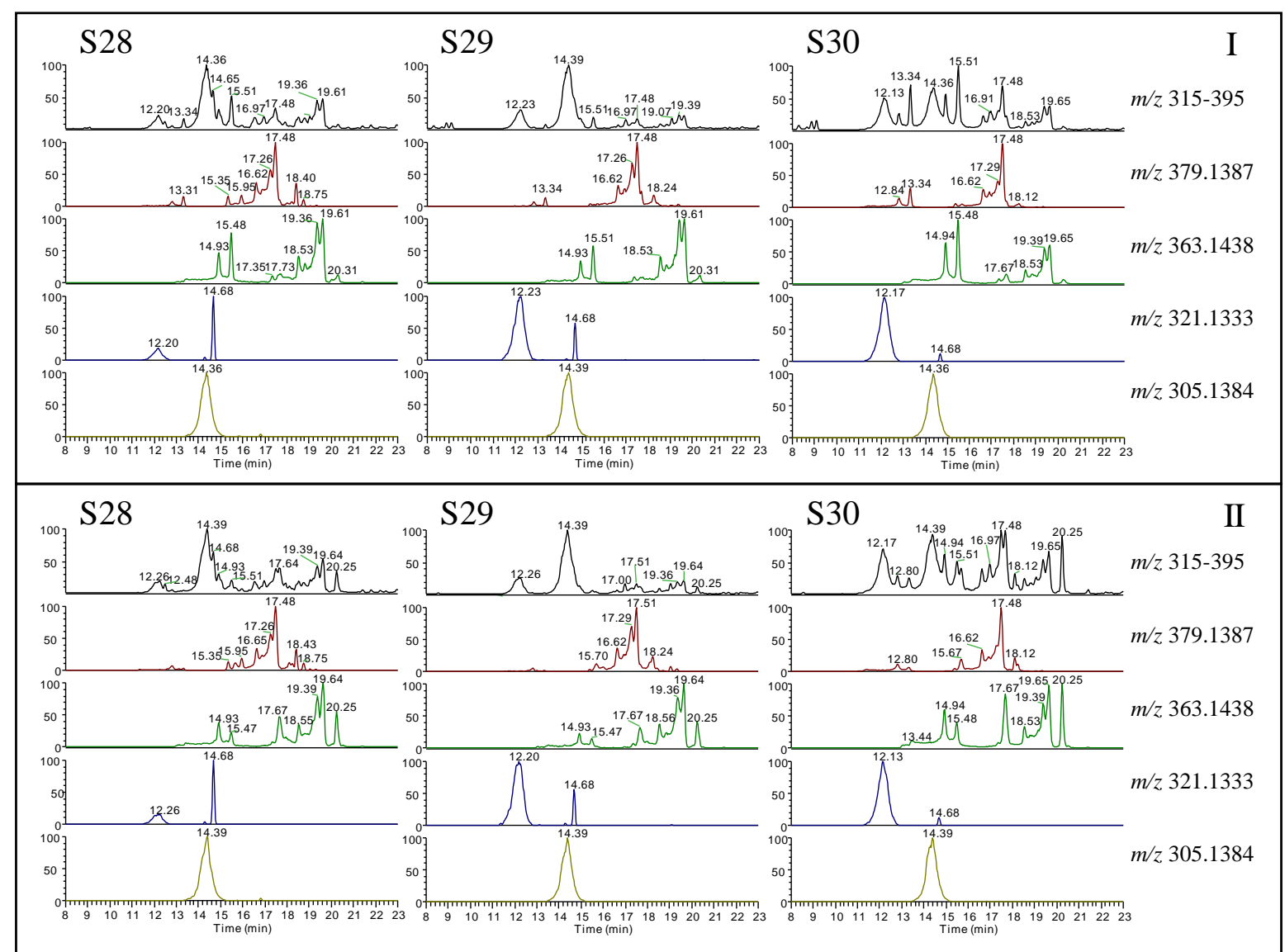

Figure 3 


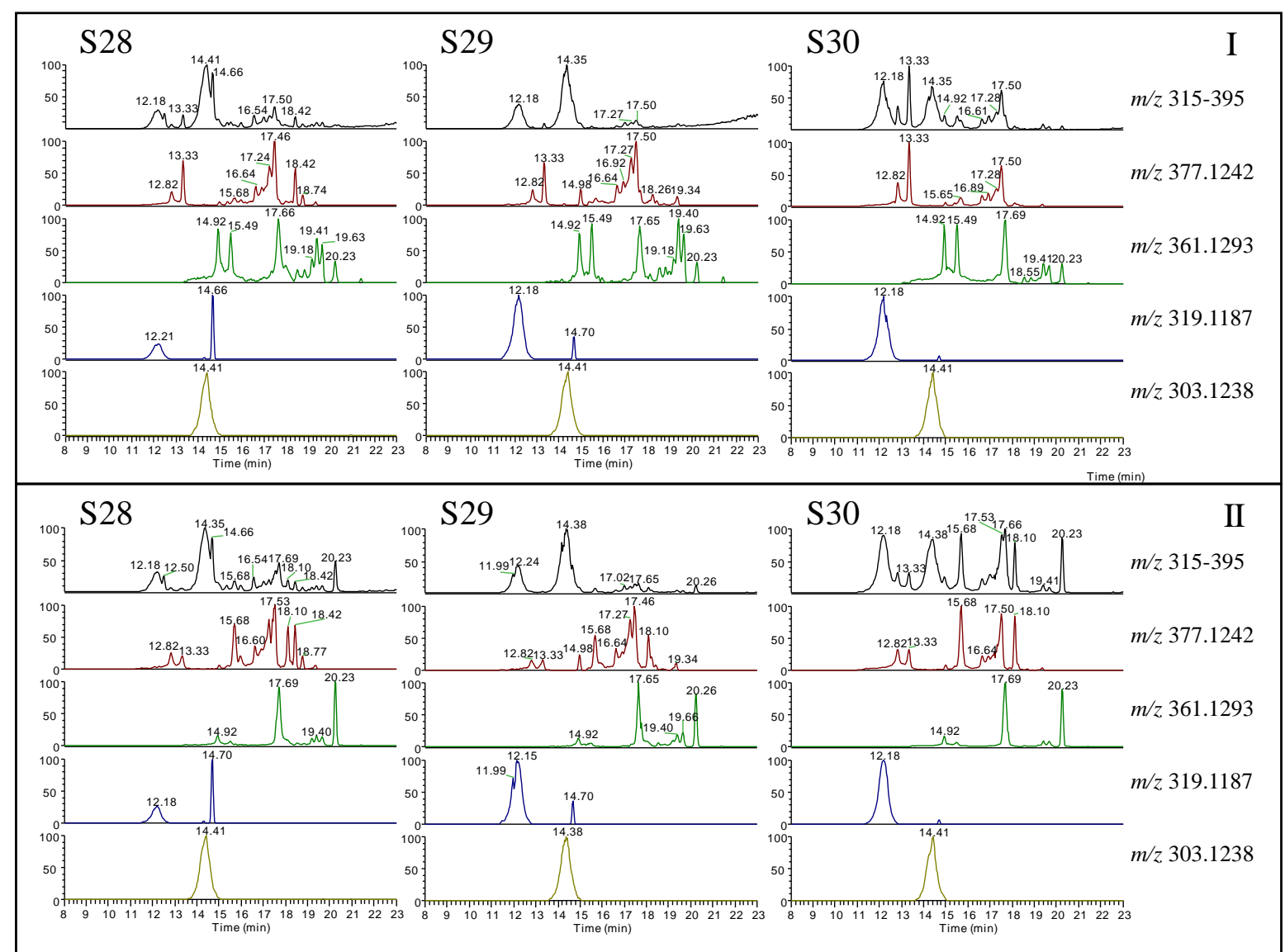

Figure 4 


\section{Figure legends}

Figure 1. TPC (mg CA/20 g oil) of samples S7-S27 estimated with the F-C assay after extraction with $\mathrm{MeOH}: \mathrm{H}_{2} \mathrm{O}, 80: 20 \mathrm{v} / \mathrm{v}(\square)$ or $\mathrm{ACN}: \mathrm{H}_{2} \mathrm{O}, 70: 30 \mathrm{v} / \mathrm{v}(\square)$ (mean value $\pm \mathrm{SD}, n=3$; *denotes statistical significant differences at $p<0.05$ )

Figure 2. HPLC-DA-FL detection profile of the polar fractions of tested oils and the baselines recorded at (a) $245 \mathrm{~nm}$, (b) $280 \mathrm{~nm}$, (c) $320 \mathrm{~nm}$, (d) $280 \mathrm{~nm} \lambda_{\text {exc }} 320 \mathrm{~nm} \lambda_{\text {em }}$ (red line: aqueous acetonitrile; black line aqueous methanol; IOC elution system containing MeOH:ACN, 1:1, v/v)

Figure 3. ESI $^{+}$full scan HRMS (orbitrap) chromatograms of polar fractions of tested oils obtained with (I) $\mathrm{MeOH}: \mathrm{H}_{2} \mathrm{O}, 80: 20$, v/v; (II) $\mathrm{ACN}: \mathrm{H}_{2} \mathrm{O}, 70: 30$, v/v. Chromatograms were obtained by selecting the mass range $m / z 315-395$ or the exact mass of the main secoiridoids: oleuropein aglycon $\left(m / z, 379.1387, \mathrm{C}_{19} \mathrm{H}_{23} \mathrm{O}_{8}\right)$, ligstroside aglycon $\left(\mathrm{m} / \mathrm{z} 363.1438, \mathrm{C}_{19} \mathrm{H}_{23} \mathrm{O}_{7}\right)$, deacetoxy oleuropein aglycon $\left(\mathrm{m} / \mathrm{z}, 321.1333, \mathrm{C}_{17} \mathrm{H}_{21} \mathrm{O}_{6}\right)$ and deacetoxy ligstroside aglycon $(\mathrm{m} / \mathrm{z} 305.1384$, $\left.\mathrm{C}_{17} \mathrm{H}_{21} \mathrm{O}_{5}\right)$. R: 70,000 (m/z 200, FWHM).

Figure 4. ESI- full scan HRMS (orbitrap) chromatograms of polar fractions of tested oils obtained with (I) $\mathrm{MeOH}: \mathrm{H}_{2} \mathrm{O}, 80: 20$, v/v; (II) $\mathrm{ACN}: \mathrm{H}_{2} \mathrm{O}, 70: 30$, v/v. Chromatograms were obtained by selecting the mass range $m / z, 315-395$ or the exact mass of the main secoiridoids: oleuropein aglycon $\left(m / z, 377.1242, \mathrm{C}_{19} \mathrm{H}_{21} \mathrm{O}_{8}\right)$, ligstroside aglycon $\left(\mathrm{m} / \mathrm{z}, 361.1293, \mathrm{C}_{19} \mathrm{H}_{21} \mathrm{O}_{7}\right)$, deacetoxy oleuropein aglycon $\left(\mathrm{m} / \mathrm{z}, 319.1187, \mathrm{C}_{17} \mathrm{H}_{19} \mathrm{O}_{6}\right)$ and deacetoxy ligstroside aglycon $(\mathrm{m} / \mathrm{z} 303.1238$, $\left.\mathrm{C}_{17} \mathrm{H}_{19} \mathrm{O}_{5}\right) . \mathrm{R}: 70,000(\mathrm{~m} / \mathrm{z}, 200, \mathrm{FWHM})$. 


\section{Supplementary material}

\section{Toward a harmonized and standardized protocol for the determination of total hydroxytyrosol and tyrosol content in VOO. Extraction solvent}

Nikolaos Nenadis ${ }^{1}$, Aspasia Mastralexi ${ }^{1}$, Maria Z. Tsimidou ${ }^{1 *}$, Stefania Vichi ${ }^{2}$, Beatriz

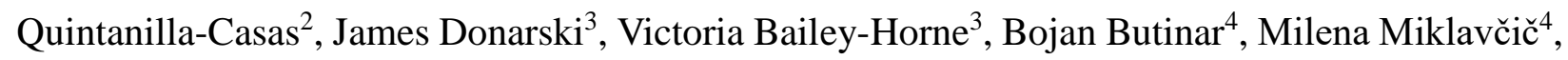
Diego-Luis García González ${ }^{5}$ Tullia Gallina Toschi ${ }^{6}$

${ }^{1}$ Laboratory of Food Chemistry and Technology, School of Chemistry, Aristotle University of Thessaloniki (AUTH), 541 24, Thessaloniki, Greece

${ }^{2}$ Food Science and Nutrition Department, XaRTA (Catalonian Reference Network on Food Technology), University of Barcelona (UB), Food and Nutrition Torribera Campus, Av. Prat de la Riba, 171. 08028, S.ta Coloma de Gramenet, Spain

${ }^{3}$ FERA Science Ltd, (FERA) Sand Hutton, York, YO41 1LZ, UK

${ }^{4}$ Laboratory of the Institute for Oliveculture, Science and Research Centre Koper (ZRS), Zelena ulica 8k, SI-6310 Izola, Slovenia

${ }^{5}$ Instituto de la Grasa (CSIC), Ctra. de Utrera, km. 1, Campus Universitario Pablo de Olavide Building 46, 41013 Seville, Spain

${ }^{6}$ Department of Agricultural and Food Sciences, Alma Mater Studiorum, University of Bologna (UNIBO), piazza Goidanich, 60, I-47521, Cesena (FC), Bologna, Italy

Corresponding author: Professor Maria Z. Tsimidou, Laboratory of Food Chemistry and Technology, School of Chemistry, Aristotle University of Thessaloniki (AUTH), 54124 , 
Thessaloniki, Greece, e-mail: tsimidou@ chem.auth.gr; Tel: +302310997796 Fax: +30 2310997847

\section{Text S1}

\section{LC-MS analysis}

(1) HRMS (UB): Chromatographic column: Halo C18 Fused-Core column $2.1 \mathrm{~mm} \times 100 \mathrm{~mm}, 2.7$ $\mu \mathrm{m}$ (Advanced Materials Technology, Wilmington, DE, USA).

Gradient: Solvent A: water 0,2\% formic acid Solvent B: $\mathrm{MeOH}$ ACN 50:50 flow rate: of 400 $\mu \mathrm{L} / \mathrm{min}$. Initial: $96 \%$ (A)-4\% (B), to $50 \%$ (B) at $20 \mathrm{~min}$; to $60 \%$ (B) at $22.5 \mathrm{~min}$, to $100 \%(\mathrm{~B})$ at $30 \mathrm{~min}, 5 \mathrm{~min}$ maintenance until $35 \mathrm{~min}$, then $96 \%$ (A)-4\% (B) at $36 \mathrm{~min}$, followed by 5 min of equilibration. Injection volume: $1 \mu \mathrm{L}$.

Mass spectrometric analysis was carried out with a Q-Exactive hybrid Orbitrap mass spectrometer (Thermo Fisher Scientific, Bremen, Germany) equipped with an electrospray source (H-ESI II). The conditions in negative ionization mode were: spray voltage $2.5 \mathrm{kV}$, capillary voltage $-25 \mathrm{~V}$, skimmer voltage $-18 \mathrm{~V}$, tube lens voltage $-110 \mathrm{~V}$. Optimized conditions in positive mode were: spray voltage $3.00 \mathrm{kV}$, capillary voltage $32.5 \mathrm{~V}$, skimmer voltage $18 \mathrm{~V}$, tube lens voltage $110 \mathrm{~V}$. In both cases, sheath gas flow rate was set at 55 arbitrary units (au), auxiliary gas flow rate was 25 au, capillary temperature was $250 \circ \mathrm{C}$, and heater temperature was $25{ }^{\circ} \mathrm{C}$. Elemental composition and structural information were in a single injection with the Orbitrap mass analyzer alternating full scan mode and all ions fragmentation (AIF) mode, in negative and positive ionization modes at a resolution power of 70,000 ( $\mathrm{m} / \mathrm{z}, 200, \mathrm{FWHM})$.

(2) HRMS (FERA): Chromatographic column: Hypersil AA-ODS 2.1 x $200 \mathrm{~mm} 5 \mu \mathrm{m}$ column (Agilent Technologies, Santa Clara, CA, USA)

Gradient: Formic acid (0.1\%) in water (aqueous) and ACN:MeOH (1:1, v/v organic) were used as the mobile phases. The gradient was initially held at $10 \%$ organic for 5 minutes with a linear gradient to $100 \%$ organic by $25 \mathrm{~min}$ before being held at $100 \%$ for 5 minutes. 
The flow rate was $0.5 \mathrm{~mL} / \mathrm{min}$ and the injection volume was $2.5 \mu \mathrm{L}$.

Mass spectrometric analysis was carried out with an Agilent Series 1200 HPLC system (Agilent Technologies, Santa Clara, CA, USA) connected to the time-of-flight mass spectrometer Agilent (TOF) 6230 (Agilent Technologies, Santa Clara, CA, USA) equipped with an electrospray interface operating in the negative ionization mode. The following operation parameters were used: capillary voltage $2,500 \mathrm{~V}$, drying gas $9 \mathrm{~L} \mathrm{~min}^{-1}$, gas temperature $350 \mathrm{C}$, nebulizer pressure $40 \mathrm{psi}$, and fragmentor voltage $150 \mathrm{~V}$. LC-TOF-MS accurate mass spectra were recorded across the range of $m / z 50-1,100$.

(3) TQd (ZRS, Science and Research Centre Koper, Laboratory of the Institute for Olive culture): Chromatographic column: Synergi $4 \mu \mathrm{m}$ Hydro - RP 80 A $(250 \times 4.6 \mathrm{~mm})$ protected with Security Guard Cartridge AQC18 4×3.0 mm (both Phenomenex, Torrance, USA).

Gradient: The one proposed by IOC ${ }^{[16]}$, phosphoric acid substituted with $0.1 \%$ formic acid in aqueous phase.

Mass spectrometric analysis was carried out with Agilent Technologies Infinity 1260 HPLC equipped with 6420 Triple Quad ESI instrument operating in negative ion mode as follows: gas temperature $300{ }^{\circ} \mathrm{C}$, gas flow $10 \mathrm{~L} \mathrm{~min}^{-1}$, nebulizer pressure 50 psi and capillary voltage $3 \mathrm{kV}$.

Detection: The main parameters for MS/MS using the MRM mode are the following:

\begin{tabular}{lllllll}
\hline Species & Precursor & Product & Frag $(\mathbf{V})$ & CE $(\mathbf{V})$ & CAcc $(\mathbf{V})$ & Polarity \\
\hline Oleacein & 319 & 123 & 110 & 18 & 7 & Negative \\
MeHAOleacein & 351 & 123 & 110 & 18 & 7 & Negative \\
DiMeAOleacein & 365 & 123 & 110 & 18 & 7 & Negative \\
Oleochantal & 303 & 137 & 110 & 18 & 7 & Negative \\
MeHAOleochantal & 335 & 137 & 110 & 18 & 7 & Negative \\
DiMeAOleochantal & 349 & 137 & 110 & 18 & 7 & Negative \\
Htyr & 153 & 123 & 100 & 20 & 7 & Negative \\
Tyr & 137 & 108 & 135 & 15 & 7 & Negative \\
\hline
\end{tabular}

MeHA:methyl hemiacetal; DiMeA: dimethyl acetal; Htyr:hydroxytyrosol; Tyr: Tyrosol 
Table S1. Solvent properties with regard to their chromatographic performance

\begin{tabular}{lllllll}
\hline Solvent & $\mathbf{E T}_{\mathbf{T}}(\mathbf{3 0})$ & $\boldsymbol{\pi}^{*}$ & $\boldsymbol{A}$ & $\boldsymbol{\beta}$ & $\boldsymbol{\varepsilon}$ & $\boldsymbol{P}^{\prime}$ \\
\hline $\mathrm{H}_{2} \mathrm{O}$ & 63.1 & 0.45 & 0.43 & 0.18 & 80 & 10.2 \\
$\mathrm{MeOH}$ & 50.4 & 0.28 & 0.43 & 0.29 & 32.7 & 5.1 \\
$\mathrm{ACN}$ & 45.6 & 0.60 & 0.15 & 0.54 & 37.5 & 5.8 \\
\hline
\end{tabular}

Snyder et al. ${ }^{[17]}$

Table S2. Peak areas of oleacein, oleocanthal and their methyl and dimethyl acetals (relative concentration expressed as \%) in the polar fraction of tested VOOs obtained with $\mathrm{ACN}: \mathrm{H}_{2} \mathrm{O}, 70: 30$, v/v or MeOH: $\mathrm{H}_{2} \mathrm{O}, 80: 20$, v/v using TQd-MRM, TOF and Orbitrap for the analysis

\section{VOOs}

\begin{tabular}{|c|c|c|c|c|c|c|}
\hline & \multicolumn{2}{|c|}{ S28 } & \multicolumn{2}{|c|}{ S29 } & \multicolumn{2}{|c|}{ S30 } \\
\hline & & \multicolumn{5}{|c|}{ Solvent mixture } \\
\hline & $\mathrm{ACN}: \mathrm{H}_{2} \mathrm{O}$ & $\mathrm{MeOH}: \mathrm{H}_{2} \mathrm{O}$, & $\mathrm{ACN}: \mathrm{H}_{2} \mathrm{O}$ & $\mathrm{MeOH}: \mathrm{H}_{2} \mathrm{O}$ & $\mathrm{ACN}: \mathrm{H}_{2} \mathrm{O}$ & $\mathrm{MeOH}: \mathrm{H}_{2} \mathrm{O}$, \\
\hline & $70: 30, \mathrm{v} / \mathrm{v}$ & $80: 20, \mathrm{v} / \mathrm{v}$ & $70: 30, \mathrm{v} / \mathrm{v}$ & $80: 20, \mathrm{v} / \mathrm{v}$ & $70: 30, \mathrm{v} / \mathrm{v}$ & $80: 20, \mathrm{v} / \mathrm{v}$ \\
\hline \multicolumn{7}{|l|}{ LC-MS facility } \\
\hline \multicolumn{7}{|l|}{ Orbitrap } \\
\hline Oleacein & 85835241 & 67561676 & 143661337 & 141667702 & 365383870 & 260374323 \\
\hline Oleocanthal & 62377440 & 55477011 & 81823864 & 72422248 & 67873804 & 58519602 \\
\hline MeHAOleacein & 79665217 & 70122635 & 132239975 & 134349456 & 333036269 & 246328731 \\
\hline DiMeAOleacein & n.d. & n.d. & n.d. & n.d. & n.d. & n.d. \\
\hline MeHAOleocanthal & 1459851 & 1237599 & 2116156 & 2505889 & 1757523 & 1496218 \\
\hline DiMeAOleocanthal & n.d. & n.d. & n.d. & n.d. & n.d. & n.d. \\
\hline Sum of artifacts as \% & 35.4 & 36.7 & 37.3 & 39.0 & 43.6 & 43.7 \\
\hline \multicolumn{7}{|l|}{ TOF } \\
\hline Oleacein & 1989372 & 1966709 & 4013786 & 3944416 & 5937125 & 5645346 \\
\hline Oleocanthal & 1661351 & 1775142 & 2872390 & 3036383 & 1776450 & 1686786 \\
\hline
\end{tabular}




\begin{tabular}{|c|c|c|c|c|c|c|}
\hline MeHAOleacein & 15102 & 9340 & 12285 & 9433 & 21889 & 22861 \\
\hline DiMeAOleacein & n.d. & n.d. & n.d. & n.d. & n.d. & n.d. \\
\hline MeHAOleocanthal & 37109 & 47615 & 60867 & 64885 & 40907 & 40127 \\
\hline DiMeAOleocanthal & n.d. & n.d. & n.d. & n.d. & n.d. & n.d. \\
\hline Sum of artifacts as \% & 1.41 & 1.50 & 1.05 & 1.05 & 0.81 & 0.85 \\
\hline \multicolumn{7}{|l|}{$T Q d$} \\
\hline Oleacein & 4062 & 5565.5 & 17117.5 & 16197.5 & 19327.5 & 23650.5 \\
\hline Oleocanthal & 20220 & 27816.5 & 79475.5 & 76655.5 & 37827 & 49767.5 \\
\hline MeHAOleacein & 552 & 495 & 1705 & 1710 & 2478.5 & 2763 \\
\hline DiMeAOleacein & 657 & 470.5 & n.d. & n.d. & n.d. & n.d. \\
\hline MeHAOleocanthal & 4568.5 & 6286 & 15810 & 15291.5 & 8727 & 11589 \\
\hline DiMeAOleocanthal & 8536.5 & 10721 & 23501.5 & 18807.5 & 14169.5 & 15936.5 \\
\hline Sum of artifacts as \% & 37.1 & 35.0 & 29.8 & 27.8 & 30.7 & 29.2 \\
\hline
\end{tabular}

MeHA: methyl hemiacetal; DiMeA: dimethyl acetal; n.d.: not detected 

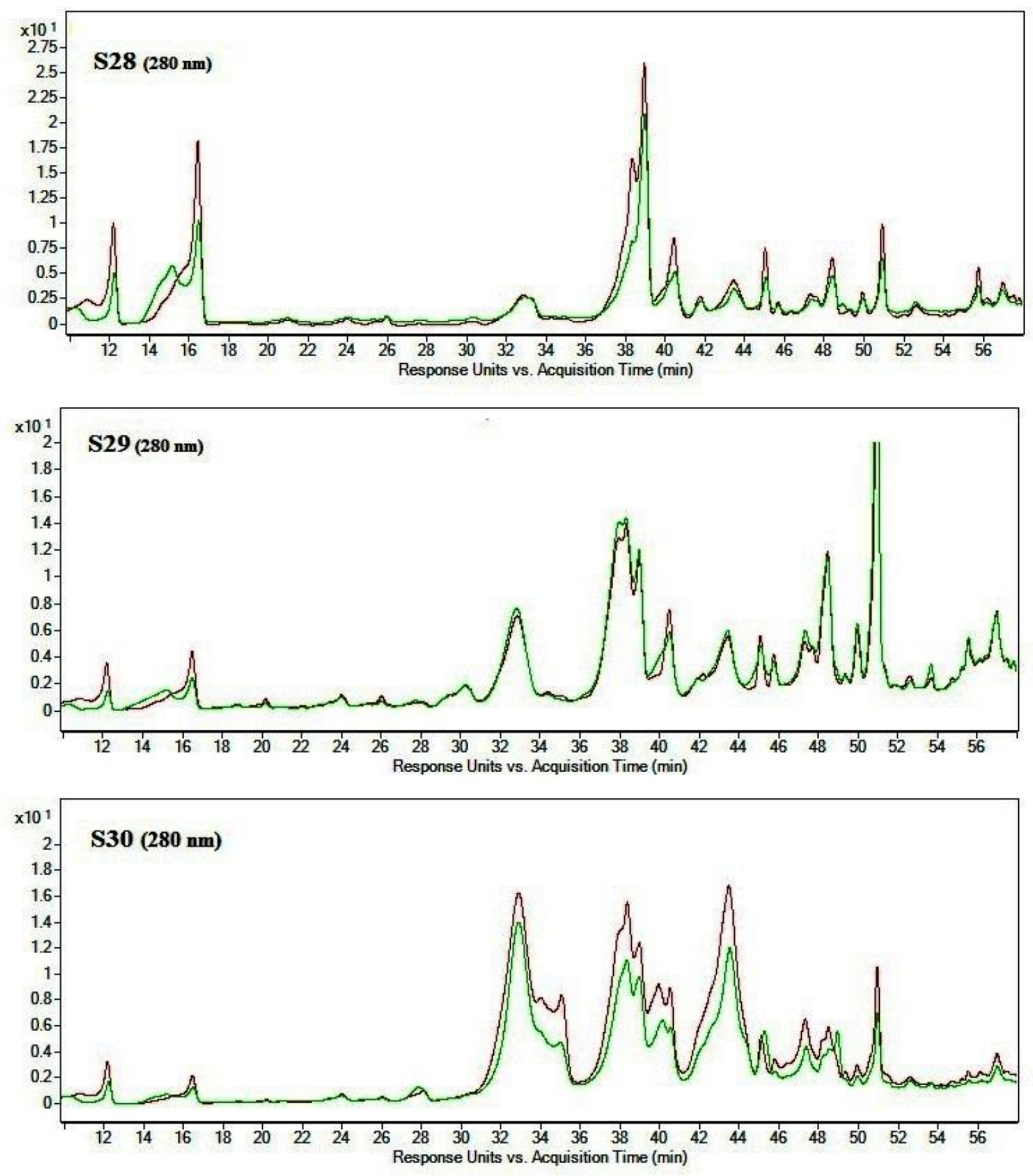

Figure S1. Chromatograms $(280 \mathrm{~nm})$ of polar fractions of tested oils obtained with $(-)$ $\mathrm{MeOH}: \mathrm{H}_{2} \mathrm{O}, 80: 20$, v/v; (-) $\mathrm{ACN}: \mathrm{H}_{2} \mathrm{O}, 70: 30$, v/v. 

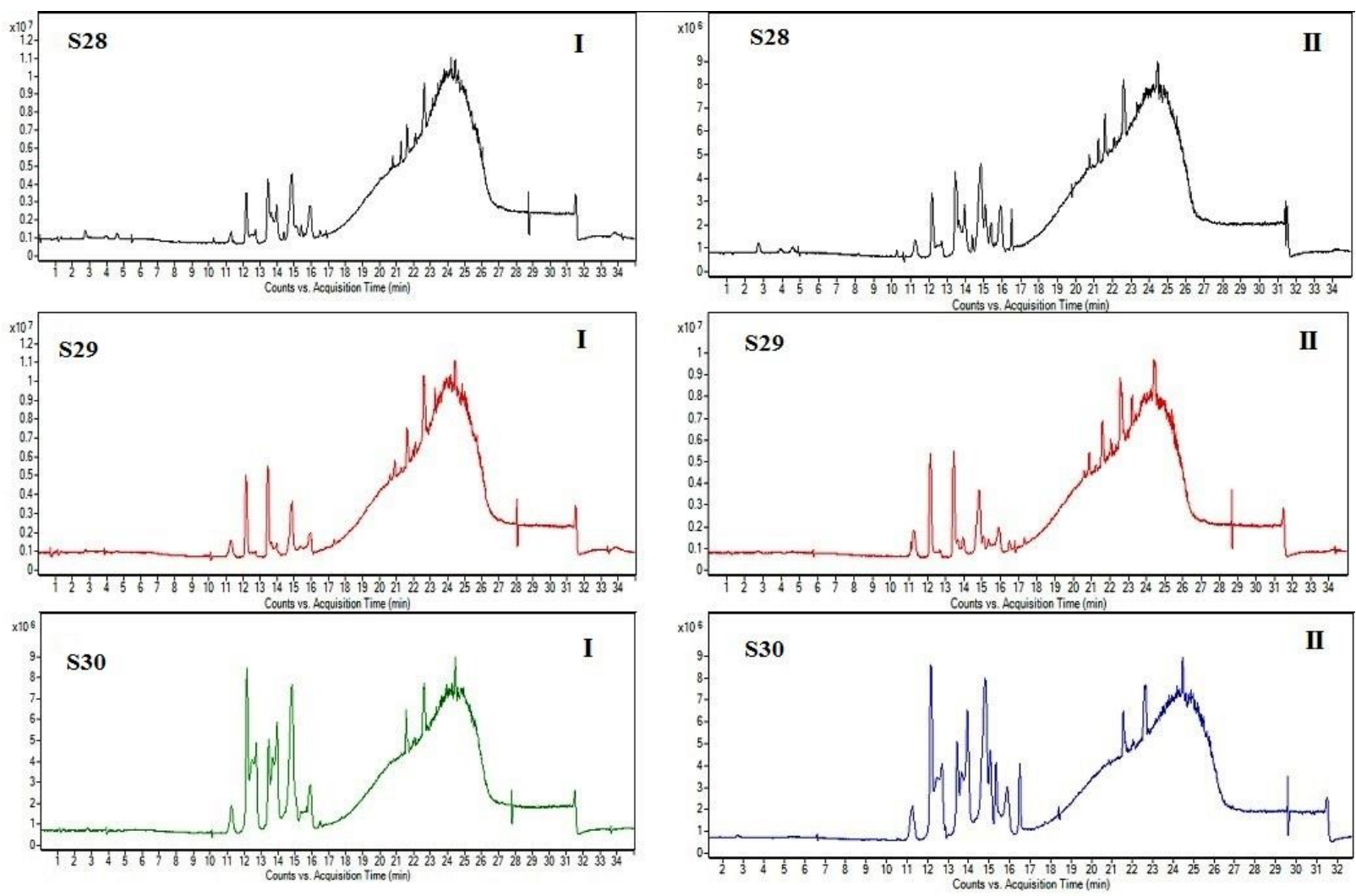

Figure S2. TOF ESI- TICs of polar fractions of tested oils obtained with (I) $\mathrm{MeOH}: \mathrm{H}_{2} \mathrm{O}, 80: 20$, v/v; (II) $\mathrm{ACN}: \mathrm{H}_{2} \mathrm{O}, 70: 30$, v/v. 